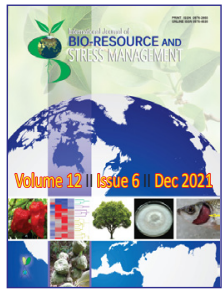

\title{
Estimation of Gene Action and Interaction for Some Quantitative Characters in Rice (Oryza sativa L.) by Generation Mean Analysis
}

\author{
Amrita Kumari*, B. K. Senapati, Anita Roy Aich and Aditya Pratap Singh
}

Dept. of Genetics and Plant Breeding, Bidhan Chandra Krishi Viswavidyalaya, Mohanpur, Nadia, West Bengal (741 252), India

\begin{tabular}{|c|c|}
\hline & Abstract \\
\hline Corresponding Author & The present investigation was conducted to understand the genetic action for \\
\hline Amrita Kumari & materials consisted of three rice varieties, i.e., Mahsuri, Bhutmuri, IR36 and F, \\
\hline e-mail: annak002900@gmail.com & $\mathrm{F}_{2,}$ and $\mathrm{F}_{3}$ populations of MahsurixBhutmuri (Cross I) and IR36×Bhutmuri (CrosS \\
\hline $\begin{array}{l}\text { Citation: Kumari et al., 2021. Estimation of Gene } \\
\text { Action and Interaction for Some Quantitative } \\
\text { Characters in Rice (Oryza sativa L.) by Generation } \\
\text { Mean Analysis. International Journal of Bio-resource } \\
\text { and Stress Management 2021, 12(6), 737-744. } \\
\text { HTTPS://DOI.ORG/10.23910/1.2021.2465. }\end{array}$ & $\begin{array}{l}\text { II). To conduct the generation mean analysis, the parents and their } F_{1}, F_{2} \text { and } \\
F_{3} \text { populations were evaluated during June to October month of Kharif } 2016 \\
\text { and Kharif } 2017 \text {. Generation mean analysis was done for eighteen quantitative } \\
\text { characters following the five parameter model. The Analysis of Variance revealed } \\
\text { significant differences among the five generations for all the characters studied. } \\
\text { The results of the scaling tests and joint scaling test revealed that the Simple }\end{array}$ \\
\hline $\begin{array}{l}\text { Copyright: (C) } 2021 \text { Kumari et al. This is an open access } \\
\text { article that permits unrestricted use, distribution and } \\
\text { reproduction in any medium after the author(s) and } \\
\text { source are credited. }\end{array}$ & $\begin{array}{l}\text { additive-dominance model was inadequate for days to } 50 \% \text { flowering, days } \\
\text { to maturity, number of panicles plant } \mathrm{t}^{-1} \text {, number of primary branches panicle }{ }^{-1} \text {, } \\
\text { number of secondary branches panicle }{ }^{-1} \text { in Cross } \mathrm{I} \text {, while it was for plant height, } \\
\text { number of tillers plant }{ }^{-1} \text {, number of panicles plant }{ }^{-1} \text {, number of grains panicle }{ }^{-1} \text {, } \\
\text { number of filled grains panicle }{ }^{-1} \text { and fertility } \% \text { in Cross } 11 \text {. Hence the present }\end{array}$ \\
\hline $\begin{array}{l}\text { Data Availability Statement: Legal restrictions are } \\
\text { imposed on the public sharing of raw data. However, } \\
\text { authors have full right to transfer or share the data in }\end{array}$ & $\begin{array}{l}\text { studies have revealed that epistasis as a basic mechanism that cannot be ignored. } \\
\text { Thus, formulating breeding policies on only main gene effects i.e. additive and } \\
\text { dominance could be misleading. }\end{array}$ \\
\hline
\end{tabular}
conditions of the original consents and the original research study. Further, access of data needs to meet whether the user complies with the ethical and legal obligations as data controllers to allow for secondary use of the data outside of the original study.

Conflict of interests: The authors have declared that no conflict of interest exists.

Keywords: Rice, gene action, GMA, joint scaling test

\section{Introduction}

Rice (Oryza sativa L.) is one of the major cereal food crops of the world, contributing nearly $73 \%$ of the total calorie intake of the population. It covers around a $20 \%$ of the total land area covered under cereals as reported by Gaballah et al., 2020. It is the most important cereal crop and primary energy source for two thirds of world's population (Khan et al., 2015). Twenty one percent of global human per capita energy and 15\% of per capita protein is provided by rice. Asia cultivates and consumes around $90 \%$ of the world's rice is cultivated and around a half of the population depends on rice for food reported by Tenorio et al., 2013. Self-sufficiency in rice production was made possible by the development of high yielding varieties. Grain yield is a complex polygenic trait in rice that is affected by the large numbers of its component traits (Liu et al., 2011). These components traits are dependent on their expression on several morphological and developmental traits, which are interrelated with each other. So, the selection and genetic improvement of these characters is the principal way of enhancing yield. In order to make the

Article History

RECEIVED on $27^{\text {th }}$ June 2021 RECEIVED in revised form on $25^{\text {th }}$ October 2021 ACCEPTED in final form on $20^{\text {th }}$ December 2021 
genetic improvement of rice, the breeding method adopted mainly depends on the nature of gene action involved in the expression of the polygenic trait. Gene action is nothing but additive and dominant effects and their interactions which are reported to be associated with a breeding value (Falconer, 1989). In such manner, generation mean analysis is an extremely valuable strategy used to estimate gene action for a polygenic trait (Kearsey and Pooni, 2004). It is a simple yet useful technique used to estimate gene effects for a polygenic trait (Kearsey and Pooni, 2004). Its greatest positive is its ability to estimate epistatic gene effects, such as additivexadditive, dominancexdominance and additivex dominance effects (Kearsey and Pooni, 2004; Viana, 2008). Using this, the gene effects of quantitative traits has been studied for various crops like in rice (Rao, 2017), Wheat (Said, (2014) pearl millet (Gaoh et al., 2020), safflower (Golkar, 2018), corn (Azizi et al., 2006), cotton (Srinivas et al., 2015, Giri et al., 2020 and Naghera et al., 2021), castor (Patel et al., 2021) and peanut (Ajay et al., 2018). Genetic analysis using generation mean analysis (GMA) has been utilized to evaluate the gene actions controlling the quantitative traits, and it gives the information on additive, dominance, and epistatic effects. Mather's individual scaling tests (Scale A, B, C, and D) and Cavalli's joint scaling test were used to detect the presence or absence of the epistatic gene interactions i.e. non-allelic gene interactions. Therefore the present investigation was carried out to estimates the gene actions, i.e., additive, dominance and epistatic effects, that control quantitative traits in rice by using five parameter model (generation mean analysis) i.e, parent $1\left(P_{1}\right)$, parent $2\left(P_{2}\right)$, first filial $\left(F_{1}\right)$, second filial $\left(F_{2}\right)$ and third filial $\left(F_{3}\right)$ generations in two rice crosses.

\section{Materials and Methods}

\subsection{Experimental material and procedure}

The experiment was carried out at the Instructional Farm, Bidhan Chandra Krishi Viswavidyalaya, Jaguli, Nadia West Bengal, India located at $22.9452^{\circ} \mathrm{N}$ Latitude and $88.5336^{\circ} \mathrm{E}$ Longitude at an altitude of $9.5 \mathrm{~m}$ above mean sea level during June to October month of Kharif 2016 and kharif 2017. The experimental materials consisted of three rice varieties, i.e., Mahsuri, Bhutmuri, IR36 and $F_{1}, F_{2}$, and $F_{3}$ populations of MahsurixBhutmuri (Cross I) and IR36×Bhutmuri (Cross II). The five populations i.e., $\mathrm{P}_{1}, \mathrm{P}_{2}, \mathrm{P}_{3}, \mathrm{~F}_{1}$ and $\mathrm{F}_{2}$ of the two crosses were grown in two separate experiments in a randomized complete blocks design with two replicates for each one. Plants were grown in 1, 2, and 3 rows each of $3 \mathrm{~m}$ length at a spacing of $30 \times 15 \mathrm{~cm}^{2}$ respectively. Observations were recorded on 10 randomly selected plants in case of parents and $F_{1}, 30$ plants of $\mathrm{F}_{2}$ and 60 plants in $\mathrm{F}_{3}$ per replication against 18 quantitative different characters such as days to $50 \%$ flowering, days to maturity, plant height $(\mathrm{cm})$, number of tillers plant ${ }^{-1}$, number of panicles plant ${ }^{-1}(\mathrm{~cm})$, panicle length, number of primary branches panicle ${ }^{-1}$, number of secondary branches panicle ${ }^{-1}$, number of grains panicle ${ }^{-1}$, number of filled grains panicle ${ }^{-1}$, fertility percentage (\%), 1000 grain weight (g), grain length $(\mathrm{mm})$, grain breadth $(\mathrm{mm})$, grain L/B ratio, straw weight $(\mathrm{g})$, harvest index (\%) and grain yield plant ${ }^{-1}(\mathrm{~g})$.

\subsection{Statistical analysis}

The analysis of variance was worked out to test the differences among genotypes by F-test. It was done by the technique of Randomized Block Design for each character according to approach upheld by Panse and Sukhatme (1967). The Scaling test was done as described by Mather (1949) and Hayman and Mather (1955) The joint scaling test as proposed by Cavalli (1952) was also applied to test the adequacy of the additivedominance model .The different components of generation mean m, d, h, i, and I were calculated following Hayman (1958) and their test of significance were calculated following the appropriate t-test.

\section{Results and Discussion}

\subsection{ANOVA}

Analysis of variance revealed significant differences among the parents, $F_{1}$ hybrids and $F_{2}$ generation against all characters studied (Table 1 and Table 2). Among the yield component in cross I, the number of secondary branches per panicle exhibited the highest significant difference. Among the yield component in cross II, the number of grains panicle ${ }^{-1}$ exhibited the highest significant difference and grain length/ breadth (L/B) exhibited the lowest significant difference in both cross I and II. It shows the presence of variation among rice genotypes used in this study.

\subsection{Scaling test, Joint scaling test and gene effects}

Generation mean analysis is a very useful technique for estimating gene effects for a polygenic character (Hayman, 1958) and determines the presence and absence of non-allelic gene interactions. Generation mean analysis can be use for the estimation of epistatic gene effects namely additivexadditive (i), additivexdominance (j), and dominancexdominance (I). The variation among the means of different generations in all the eighteen characters studied suggesting the usefulness of the estimation of additive, dominance and epistatic interaction. The $C$ and $D$ scaling test for all the characters in the two crosses showed that at least one or both were found significant indicating the presence of non-allelic interaction. Both the crosses exhibiting non-allelic interaction for the inheritance of almost all the traits studied. However, the characters like number of grains panicle ${ }^{-1}$ and harvest index (\%) in cross I and 1000 grains weight (g) in cross II showed non-significant values for both $C$ and $D$ scales (from table 2) indicating the non-interacting mode of inheritance. Similar finding were reported by Yadav et al. (2013) for number of grains per panicle and Jondhale et al. (2018) for harvest index (\%). The present study shows that significant additive and epistatic effects exist in this population. Although their presence may vary from the Cross to Cross, the estimated values of joint scaling test and the five-parameter model are presented in Table 3. 


\begin{tabular}{|c|c|c|c|c|c|c|}
\hline \multirow[t]{2}{*}{ SI. No. } & \multirow[t]{2}{*}{ Characters } & \multicolumn{3}{|c|}{ Mean sum of square } & \multirow[t]{2}{*}{ SEm \pm} & \multirow{2}{*}{$\begin{array}{c}C D \\
(p=0.05)\end{array}$} \\
\hline & & $\begin{array}{c}\text { Replication } \\
\text { (d.f. 3) }\end{array}$ & $\begin{array}{c}\text { Generation } \\
\text { (d.f. 4) }\end{array}$ & $\begin{array}{c}\text { Error } \\
\text { (d.f. 12) } \\
\end{array}$ & & \\
\hline 1. & Days to $50 \%$ flowering & 5.12 & $537.58^{* * *}$ & 12 & 2.39 & 5.20 \\
\hline 2. & Days to maturity & 3.0 & $473.33^{* * *}$ & 4.13 & 1.44 & 3.13 \\
\hline 3. & Plant height $(\mathrm{cm})$ & 3.23 & $118.11^{* * *}$ & 4.84 & 1.56 & 3.39 \\
\hline 4. & No. of tillers plant ${ }^{-1}$ & 0.15 & $74.89^{* * *}$ & 1.29 & 0.8 & 1.75 \\
\hline 5. & No. of panicles plant ${ }^{-1}$ & 3.0 & $115.36^{* * *}$ & 1.66 & 0.91 & 1.99 \\
\hline 6. & Panicle length(cm) & 2.15 & $29.13^{* *}$ & 4.90 & 1.57 & 3.41 \\
\hline 7. & No. of primary branches panicle ${ }^{-1}$ & 0.48 & $37.66^{* * *}$ & 1.09 & 0.74 & 1.61 \\
\hline 8. & No. of secondary braches panicle ${ }^{-1}$ & 5.90 & $1301.27^{* * *}$ & 10.83 & 2.33 & 5.07 \\
\hline 9. & No. of grains panicle ${ }^{-1}$ & 20.01 & $847.44^{* * *}$ & 66.30 & 5.76 & 12.55 \\
\hline 10. & No. of filled grains panicle ${ }^{-1}$ & 18.75 & $910.18^{* * *}$ & 18.14 & 3.01 & 6.56 \\
\hline 11. & Fertility percentage (\%) & 5.32 & $48.56^{*}$ & 7.61 & 1.95 & 4.25 \\
\hline 12. & 1000 grain weight (g) & 1.79 & $22.07^{* *}$ & 3.87 & 1.39 & 3.03 \\
\hline 13. & Grain length (mm) & 0.05 & $0.89^{*}$ & 0.18 & 0.30 & 0.66 \\
\hline 14. & Grain breadth (mm) & 0.08 & $0.67^{* * *}$ & 0.03 & 0.12 & 0.27 \\
\hline 15. & Grain length/breadth (L/B) ratio & 0.13 & $0.40^{*}$ & 0.08 & 0.20 & 0.45 \\
\hline 16. & Straw weight (g) & 2.06 & $62.06^{* * *}$ & 5.59 & 1.67 & 3.64 \\
\hline 17. & Harvest index (\%) & $23.18^{*}$ & $37.89^{* * *}$ & 2.96 & 1.22 & 2.65 \\
\hline 18. & Grain yield plant $^{-1}(\mathrm{~g})$ & 19.73 & $141.15^{* * *}$ & 6.56 & 1.81 & 3.94 \\
\hline
\end{tabular}

*Significant at $(p=0.05) ; * *(p=0.01),{ }^{* *}(p=0.001)$, SEm: Standard Error of mean; CD: Critical Difference

\begin{tabular}{|c|c|c|c|c|c|}
\hline \multirow[t]{2}{*}{ Characters } & \multicolumn{3}{|c|}{ Mean sum of square } & \multirow[t]{2}{*}{ SEm \pm} & \multirow{2}{*}{$\begin{array}{c}C D \\
(p=0.05)\end{array}$} \\
\hline & $\begin{array}{l}\text { Replication } \\
\text { (d.f. 3) }\end{array}$ & $\begin{array}{l}\text { Generation } \\
\text { (d.f. 4) }\end{array}$ & $\begin{array}{c}\text { Error } \\
\text { (d.f. 12) }\end{array}$ & & \\
\hline Days to $50 \%$ flowering & 25.73 & $305.30^{* * *}$ & 27.73 & 3.72 & 8.11 \\
\hline Days to maturity & 28.18 & $415.88^{* * *}$ & 16.64 & 2.88 & 6.28 \\
\hline Plant height(cm) & 3.61 & $1073.75^{* * *}$ & 8.39 & 2.05 & 4.46 \\
\hline No. of tillers plant ${ }^{-1}$ & 1.25 & $488.16^{* * *}$ & 0.80 & 0.63 & 1.38 \\
\hline No. of panicles plant ${ }^{-1}$ & 1.93 & $126.40^{* * *}$ & 4.62 & 1.52 & 3.31 \\
\hline Panicle length $(\mathrm{cm})$ & $6.68^{*}$ & $10.70^{* *}$ & 1.56 & 0.88 & 1.92 \\
\hline No. of primary branches panicle ${ }^{-1}$ & 1.99 & $9.21^{* *}$ & 1.60 & 0.90 & 1.95 \\
\hline No. of secondary braches panicle $e^{-1}$ & 6.41 & $51.73^{* *}$ & 8.72 & 2.09 & 4.55 \\
\hline No. of grains panicle ${ }^{-1}$ & 8.25 & $4936.73^{* * *}$ & 12.01 & 2.45 & 5.34 \\
\hline No. of filled grains panicle ${ }^{-1}$ & 1.95 & $3921.83^{* * *}$ & 14.72 & 2.71 & 5.91 \\
\hline Fertility percentage (\%) & 2.36 & $121.66^{* * *}$ & 3.24 & 1.27 & 2.77 \\
\hline 1000 grain weight (g) & $7.30^{*}$ & $7.66^{*}$ & 1.67 & 0.91 & 1.99 \\
\hline Grain length (mm) & 0.05 & $0.55^{* * *}$ & 0.05 & 0.15 & 0.33 \\
\hline Grain breadth (mm) & 0.07 & $0.39^{*}$ & 0.06 & 0.18 & 0.39 \\
\hline Grain length/breadth (L/B) ratio & 0.09 & $0.37^{*}$ & 0.31 & 0.39 & 0.81 \\
\hline
\end{tabular}




\begin{tabular}{|c|c|c|c|c|c|}
\hline \multirow[t]{2}{*}{ Characters } & \multicolumn{3}{|c|}{ Mean sum of square } & \multirow[t]{2}{*}{ SEm \pm} & \multirow{2}{*}{$\begin{array}{c}C D \\
(p=0.05)\end{array}$} \\
\hline & $\begin{array}{l}\text { Replication } \\
\text { (d.f. 3) }\end{array}$ & $\begin{array}{c}\text { Generation } \\
\text { (d.f. 4) }\end{array}$ & $\begin{array}{c}\text { Error } \\
\text { (d.f. 12) }\end{array}$ & & \\
\hline Straw weight (g) & 2.95 & $24.52^{* * *}$ & 1.94 & 0.98 & 2.14 \\
\hline Harvest index (\%) & 14.02 & $29.93^{*}$ & 9.06 & 2.13 & 4.64 \\
\hline Grain yield plant ${ }^{-1}(\mathrm{~g})$ & 8.31 & $41.61^{*}$ & 8.46 & 2.06 & 4.48 \\
\hline
\end{tabular}

*Significant at $(p=0.05) ; * *(p=0.01), * *(p=0.001)$, SEm: Standard Error of mean; CD: Critical Difference

Table 3: Different scales for yield components and component of generation means for different characters in cross I and cross II as suggested by Hayman (1958)

\begin{tabular}{|c|c|c|c|c|c|c|c|c|c|c|c|c|}
\hline \multirow[t]{2}{*}{$\mathrm{C}^{*}$} & \multicolumn{2}{|c|}{ Scaling test } & \multicolumn{3}{|c|}{ Three parameter mode } & \multirow{2}{*}{$\begin{array}{c}\begin{array}{c}\text { Joint } \\
\text { scaling } \\
\text { test }\end{array} \\
\left(\chi^{2}\right)\end{array}$} & \multicolumn{5}{|c|}{ Five parameter model } & \multirow{2}{*}{$\begin{array}{l}\text { Epis- } \\
\text { tasis }\end{array}$} \\
\hline & $\mathrm{C}$ & $\mathrm{D}$ & $\mathrm{m}$ & $\mathrm{d}$ & $\mathrm{h}$ & & $\mathrm{m}$ & $d$ & $\mathrm{~h}$ & $\mathrm{i}$ & I & \\
\hline \multicolumn{13}{|c|}{ Days to $50 \%$ flowering } \\
\hline I & 1.25 & $11.75^{* * *}$ & - & - & - & $8.82^{*}$ & $\begin{array}{c}117^{* * *} \pm \\
0.43\end{array}$ & $\begin{array}{c}15.63^{* * *} \\
\pm 0.25\end{array}$ & $\begin{array}{c}0.50 \pm \\
1.36\end{array}$ & $\begin{array}{c}23.63^{* * *} \\
\pm 1.53\end{array}$ & $\begin{array}{c}14^{* *} \pm \\
4.93\end{array}$ & $\mathrm{C}$ \\
\hline II & -9 & $-14^{* *}$ & $\begin{array}{c}79.18^{* * *} \\
\pm 1.17\end{array}$ & $\begin{array}{l}-12 \pm \\
0.79\end{array}$ & $\begin{array}{l}6.5 \pm \\
1.81\end{array}$ & 0.45 & - & - & - & - & - & - \\
\hline \multicolumn{13}{|c|}{ Days to maturity } \\
\hline I & $-4^{*}$ & $15.00^{* * *}$ & - & - & - & $55.68^{* * *}$ & $\begin{array}{c}147.5^{* * *} \pm \\
0.34\end{array}$ & $\begin{array}{l}14.75^{* *} \\
\pm 0.37\end{array}$ & $\begin{array}{c}-3.67^{* * *} \\
\pm 0.83\end{array}$ & $\begin{array}{c}18.83^{* * *} \\
\pm 1.16\end{array}$ & $\begin{array}{c}25.33^{* * *} \\
\pm 1.16\end{array}$ & $\mathrm{D}$ \\
\hline II & -1.25 & -4.75 & $\begin{array}{c}116.25^{* * *} \\
\pm 0.52\end{array}$ & $\begin{array}{l}-13.89 \\
\pm 0.87\end{array}$ & $\begin{array}{c}10.73^{* * *} \\
\pm 0.70\end{array}$ & 2.42 & - & - & - & - & - & - \\
\hline \multicolumn{13}{|c|}{ Plant height $(\mathrm{cm})$} \\
\hline I & $-27.25^{* * *}$ & $-25.50^{* * *}$ & $\begin{array}{c}126.56^{* * *} \\
\pm 0.32\end{array}$ & $\begin{array}{l}2.88^{* *} \\
\pm 0.41\end{array}$ & $\begin{array}{l}17.96^{* * *} \\
\pm 0.547\end{array}$ & 1.04 & - & - & - & - & - & - \\
\hline II & $28.01^{* * *}$ & $-17.49^{* * *}$ & - & - & - & $125.62^{* * *}$ & $\begin{array}{c}130.75^{* * *} \\
\pm 0.23\end{array}$ & $\begin{array}{c}-20.63^{* * *} \\
\pm 0.55\end{array}$ & $\begin{array}{c}30.83^{* * *} \\
\pm 0.85\end{array}$ & $\begin{array}{c}-24.93^{* * *} \\
\pm 1.25\end{array}$ & $\begin{array}{c}-60.67^{* * *} \\
\pm 2.52\end{array}$ & $\mathrm{D}$ \\
\hline \multicolumn{13}{|c|}{ Number of tillers Plant ${ }^{-1}$} \\
\hline I & $-10.37^{* * *}$ & -1.13 & $\begin{array}{c}19.88^{* * *} \\
\pm 0.25\end{array}$ & $\begin{array}{l}-2.19 \\
\pm 0.20\end{array}$ & $\begin{array}{c}0.88 \pm \\
1.21\end{array}$ & 3.63 & - & - & - & - & - & - \\
\hline II & $-21.88^{* * *}$ & $14.38^{* * *}$ & - & - & - & $91.81^{* * *}$ & $\begin{array}{c}24.37^{* * *} \\
\pm 0.21\end{array}$ & $\begin{array}{c}-5.43^{* * *} \pm \\
0.14\end{array}$ & $\begin{array}{c}12.08^{* * *} \\
\pm 0.69\end{array}$ & $\begin{array}{c}-24.10^{* * *} \\
\pm 0.68\end{array}$ & $\begin{array}{c}48.33^{* * *} \\
\pm 1.99\end{array}$ & C \\
\hline \multicolumn{13}{|c|}{ Number of panicles plant ${ }^{-1}$} \\
\hline I & $27.90^{* * *}$ & $15.40^{* * *}$ & - & - & - & $13.59^{* * *}$ & $\begin{array}{c}29.88^{* * *} \\
\pm 0.21\end{array}$ & $\begin{array}{c}-2.80^{* * *} \\
\pm 0.13\end{array}$ & $\begin{array}{c}1.33 \pm \\
1.33\end{array}$ & $\begin{array}{c}-11.21^{* * *} \\
\pm 1.10\end{array}$ & $\begin{array}{c}-16.66^{* * *} \\
\pm 3.07\end{array}$ & $\mathrm{D}$ \\
\hline II & -0.6 & $8.9^{* *}$ & - & - & - & $0.08^{* *}$ & $\begin{array}{c}25.63^{* * *} \\
\pm 0.22\end{array}$ & $\begin{array}{l}-2.80^{* * *} \\
\pm 0.18\end{array}$ & $\begin{array}{c}6.67^{* * *} \pm \\
1.89\end{array}$ & $\begin{array}{c}-11.63^{* * *} \\
\pm 1.46\end{array}$ & $\begin{array}{c}12.67^{* *} \\
\pm 4.18\end{array}$ & $\mathrm{C}$ \\
\hline \multicolumn{13}{|c|}{ Panicle length (cm) } \\
\hline I & $-11.25^{* * *}$ & $-11.50^{* * *}$ & $\begin{array}{c}21.80^{* * *} \\
\pm 0.44\end{array}$ & $\begin{array}{l}2.13^{* * *} \\
\pm 0.13\end{array}$ & $\begin{array}{c}3.33 \pm \\
0.66\end{array}$ & 0.01 & - & - & - & & & \\
\hline II & $\frac{-}{1.5}$ & $\begin{array}{c}- \\
-5.75^{* * *}\end{array}$ & $\begin{array}{c}- \\
22.52^{* * *} \\
\pm 0.39\end{array}$ & $\begin{array}{c}-1.88 \pm \\
0.24\end{array}$ & $\begin{array}{c}2.64 \pm \\
0.54\end{array}$ & 8.8 & - & - & - & - & - & - \\
\hline
\end{tabular}

$C^{*}:$ Crosses

Table 3: Continue... 


\begin{tabular}{|c|c|c|c|c|c|c|c|c|c|c|c|c|}
\hline \multirow[t]{2}{*}{$\mathrm{C}^{*}$} & \multicolumn{2}{|c|}{ Scaling test } & \multicolumn{3}{|c|}{ Three parameter mode } & \multirow{2}{*}{$\begin{array}{c}\text { Joint } \\
\text { scaling } \\
\text { test } \\
\left(\chi^{2}\right)\end{array}$} & \multicolumn{5}{|c|}{ Five parameter model } & \multirow{2}{*}{$\begin{array}{l}\text { Epis- } \\
\text { tasis }\end{array}$} \\
\hline & $\mathrm{C}$ & $\mathrm{D}$ & $\mathrm{m}$ & $\mathrm{d}$ & $\mathrm{h}$ & & $\mathrm{m}$ & $\mathrm{d}$ & $\mathrm{h}$ & i & 1 & \\
\hline \multicolumn{13}{|c|}{ Number of primary branches panicle ${ }^{-1}$} \\
\hline I & $7.13^{* * *}$ & $-6.12^{* * *}$ & - & - & - & $21.76^{* * *}$ & $\begin{array}{c}17.25^{* * *} \\
\pm 0.19\end{array}$ & $\begin{array}{l}1.06^{* * *} \\
\pm 0.11\end{array}$ & $\begin{array}{c}11.58^{* * *} \\
\pm 0.90\end{array}$ & $\begin{array}{c}7.39^{* * *} \pm \\
0.79\end{array}$ & $\begin{array}{c}-17.67^{* * *} \\
\pm 2.26\end{array}$ & $\mathrm{D}$ \\
\hline II & $-8.40^{* * *}$ & $-7.79^{* * *}$ & $\begin{array}{l}6.88^{* * *} \\
\pm 0.144\end{array}$ & $\begin{array}{l}-0.38 \\
\pm 0.22\end{array}$ & $\begin{array}{l}2.96^{*} \\
\pm 1.81\end{array}$ & 3.14 & - & - & - & - & - & - \\
\hline \multicolumn{13}{|c|}{ Number of secondary braches panicle ${ }^{-1}$} \\
\hline I & $-8.13^{*}$ & $79.13^{* * *}$ & - & - & - & $308.58^{* * *}$ & $\begin{array}{c}39.13^{* * *} \\
\pm 0.61\end{array}$ & $\begin{array}{l}7.81^{* * *} \\
\pm 0.24\end{array}$ & $\begin{array}{c}-15.41^{* * *} \\
\pm 1.77\end{array}$ & $\begin{array}{c}-38.47^{* * *} \\
\pm 1.93\end{array}$ & $\begin{array}{c}116.3^{* * *} \\
\pm 6.06\end{array}$ & $\mathrm{D}$ \\
\hline II & $-11.13^{*}$ & $-24.13^{* * *}$ & $\begin{array}{c}27.47^{* * *} \\
\pm 0.45\end{array}$ & $\begin{array}{l}2.063^{*} \\
\pm 0.27\end{array}$ & $\begin{array}{c}10.66^{* * *} \\
\pm 0.48\end{array}$ & 3.74 & - & - & - & - & - & - \\
\hline \multicolumn{13}{|c|}{ Number of grains panicle ${ }^{-1}$} \\
\hline I & 4.88 & 7.88 & $\begin{array}{c}95.17^{* * *} \\
\pm 0.56\end{array}$ & $\begin{array}{c}18.61^{* * *} \\
\pm 0.59\end{array}$ & $\begin{array}{l}15.23^{*} \\
\pm 1.75\end{array}$ & 3.42 & - & - & - & - & - & - \\
\hline II & $16^{* * *}$ & $-143^{* * *}$ & - & - & - & $1109.33^{* * *}$ & $\begin{array}{c}34.87^{* * *} \\
\pm 1.09\end{array}$ & $\begin{array}{l}2.06^{* * *} \\
\pm 0.27\end{array}$ & $\begin{array}{c}15.17^{* * *} \\
\pm 2.38\end{array}$ & $\begin{array}{c}18.35^{* * *} \\
\pm 2.80\end{array}$ & $\begin{array}{c}-17.33 \pm \\
8.96\end{array}$ & $\mathrm{D}$ \\
\hline \multicolumn{13}{|c|}{ Number of filled grains panicle ${ }^{-1}$} \\
\hline I & -4.13 & $17.88^{* *}$ & $\begin{array}{c}61.17^{* * *} \\
\pm 0.41\end{array}$ & $\begin{array}{c}14.41^{* * *} \\
\pm 0.43\end{array}$ & $\begin{array}{c}26.76^{* * *} \\
\pm 0.60\end{array}$ & 0.15 & - & - & - & - & - & - \\
\hline II & -4.88 & $-120.38^{* * *}$ & - & - & - & $496.34^{* * *}$ & $\begin{array}{c}133.50^{* * *} \\
\pm 0.74\end{array}$ & $\begin{array}{l}40.63^{* * *} \\
\pm 0.56\end{array}$ & $\begin{array}{c}123.50^{* * *} \\
\pm 1.78\end{array}$ & $\begin{array}{c}179.25^{* * *} \\
\pm 2.14\end{array}$ & $\begin{array}{l}-212^{* * *} \\
\pm 6.36\end{array}$ & $\mathrm{D}$ \\
\hline \multicolumn{13}{|c|}{ Number of panicles plant ${ }^{-1}$} \\
\hline I & $27.90^{* * *}$ & $15.40^{* * *}$ & - & - & - & $13.59^{* * *}$ & $\begin{array}{c}29.88^{* * *} \\
\pm 0.21\end{array}$ & $\begin{array}{c}-2.80^{* * *} \\
\pm 0.13\end{array}$ & $\begin{array}{c}1.33 \pm \\
1.33\end{array}$ & $\begin{array}{c}-11.21^{* * *} \\
\pm 1.10\end{array}$ & $\begin{array}{c}-16.66^{* * *} \\
\pm 3.07\end{array}$ & $\mathrm{D}$ \\
\hline II & -0.6 & $8.9^{* *}$ & - & - & - & $0.08^{* *}$ & $\begin{array}{c}25.63^{* * *} \\
\pm 0.22\end{array}$ & $\begin{array}{l}-2.80^{* * *} \\
\pm 0.18\end{array}$ & $\begin{array}{c}6.67^{* * *} \pm \\
1.89\end{array}$ & $\begin{array}{c}-11.63^{* * *} \\
\pm 1.46\end{array}$ & $\begin{array}{l}12.67^{* *} \\
\pm 4.18\end{array}$ & $\mathrm{C}$ \\
\hline \multicolumn{13}{|c|}{ Fertility percent (\%) } \\
\hline I & $9.52^{* * *}$ & $15.48^{* * *}$ & $\begin{array}{c}66.62^{* * *} \\
\pm 0.49\end{array}$ & $\begin{array}{l}-0.89 \\
\pm 0.45\end{array}$ & $\begin{array}{l}-0.13 \\
\pm 0.71\end{array}$ & 6.69 & - & - & - & - & - & - \\
\hline II & $4.74^{* * *}$ & $-11.57^{* * *}$ & - & - & - & $99.06^{* * *}$ & $\begin{array}{c}67.60^{* * *} \\
\pm 0.22\end{array}$ & $\begin{array}{l}7.29^{* * *} \\
\pm 0.36\end{array}$ & $\begin{array}{c}4.32^{* * *} \pm \\
0.62\end{array}$ & $\begin{array}{c}23.09^{* * *} \\
\pm 0.99\end{array}$ & $\begin{array}{c}-21.75^{* * *} \\
\pm 1.98\end{array}$ & $\mathrm{D}$ \\
\hline & 0 Grain v & eight (g) & & & & & & & & & & \\
\hline I & $-6.97^{* * *}$ & 1.75 & $\begin{array}{c}19.77^{* * *} \\
\pm 0.23\end{array}$ & $\begin{array}{c}-3.04 \\
\pm 0.233\end{array}$ & $\begin{array}{c}-7.89 \pm \\
1.54\end{array}$ & 1.28 & - & - & - & - & - & - \\
\hline II & -1.93 & -0.27 & $\begin{array}{c}18.74^{* * *} \\
\pm 0.22\end{array}$ & $\begin{array}{l}-1.52 \\
\pm 0.28\end{array}$ & $\begin{array}{c}-1.47 \pm \\
0.34\end{array}$ & 2.49 & - & - & - & - & - & - \\
\hline
\end{tabular}

C*: Crosses

Table 3: Continue... 


\begin{tabular}{|c|c|c|c|c|c|c|c|c|c|c|c|c|}
\hline \multirow[t]{2}{*}{ Crosses } & \multicolumn{2}{|c|}{ Scaling test } & \multicolumn{3}{|c|}{ Three parameter mode } & \multirow{2}{*}{$\begin{array}{c}\text { Joint } \\
\text { scaling } \\
\text { test }\end{array}$} & \multicolumn{5}{|c|}{ Five parameter model } & \multirow[t]{2}{*}{ Epistasis } \\
\hline & $\mathrm{C}$ & $\mathrm{D}$ & $\mathrm{m}$ & d & $\mathrm{h}$ & & $\mathrm{m}$ & $d$ & $\mathrm{~h}$ & $\mathrm{i}$ & 1 & \\
\hline \multicolumn{13}{|c|}{ Grain length $(\mathrm{mm})$} \\
\hline 1 & $-1.57^{* * *}$ & $-1.46^{* * *}$ & $7.28^{* * *} \pm 0.09$ & $-0.46 \pm 0.04$ & $0.26 \pm 0.18$ & 2.27 & - & - & - & - & - & - \\
\hline ॥ & $-1.38^{* * *}$ & $-1.53^{* * *}$ & $7.18^{* * *} \pm 0.05$ & $-0.04 \pm 0.03$ & $0.09 \pm 0.71$ & 3.54 & - & - & - & - & - & - \\
\hline \multicolumn{13}{|c|}{ Grain breadth $(\mathrm{mm})$} \\
\hline 1 & $-1.65^{* * *}$ & $-1.60^{* * *}$ & $2.01^{* * *} \pm 0.05$ & $-0.4 \pm 0.03$ & $0.64^{* *} \pm 0.05$ & 0.027 & - & - & - & - & - & - \\
\hline II & $-0.73^{*}$ & $-1.23^{* * *}$ & $2.35^{* * *} \pm 0.07$ & $0.34^{* *} \pm 0.03$ & $0.23 \pm 0.07$ & 1.17 & - & - & - & - & - & - \\
\hline \multicolumn{13}{|c|}{ Grain length/ breadth (L/B) ratio } \\
\hline 1 & $1.21^{* * *}$ & $1.20^{* * *}$ & $3.57^{* * *} \pm 0.06$ & $0.32 * \pm 0.045$ & $-0.72 \pm 0.07$ & 0 & - & - & - & - & - & - \\
\hline II & 0.57 & $1.03^{* * *}$ & $3.24^{* * *} \pm 0.08$ & $-0.36 \pm 0.03$ & $-0.62 \pm 0.14$ & 0.97 & - & - & - & - & - & - \\
\hline \multicolumn{13}{|c|}{ Straw weight (g) } \\
\hline 1 & $12.23^{* * *}$ & $10.92^{* * *}$ & $47.29^{* * *} \pm 0.45$ & $3.84^{* *} \pm 0.38$ & $-10.57 \pm 0.72$ & 0.3 & - & - & - & - & - & - \\
\hline ॥ & $4.32^{* * *}$ & 2.59 & $37.25^{* * *} \pm 0.16$ & $3.31^{* * *} \pm 0.06$ & $-0.84 \pm 3.14$ & 0.071 & - & - & - & - & - & - \\
\hline \multicolumn{13}{|c|}{ Harvest index percent (\%) } \\
\hline 1 & -3.82 & 0.96 & $40.65^{* * *} \pm 0.34$ & $3.39^{* *} \pm 0.39$ & $4.79 \pm 0.62$ & 2.93 & - & - & - & - & - & - \\
\hline II & -1.18 & $7.89^{*}$ & $40.34^{* * *} \pm 0.33$ & $0.86 \pm 0.51$ & $-6.64 \pm 5.56$ & 4.32 & - & - & - & - & - & - \\
\hline \multicolumn{13}{|c|}{ Grain yield plant $^{-1}(\mathrm{~g})$} \\
\hline 1 & $8.01^{* *}$ & $10.17^{* * *}$ & $32.28^{* * *} \pm 0.75$ & $7.86^{* * *} \pm 0.43$ & $-1.32 \pm 1.23$ & 1.44 & - & - & - & - & - & - \\
\hline II & 0.75 & $9.67^{* *}$ & $27.72^{* * *} \pm 1.34$ & $2.94^{*} \pm 0.48$ & $-8.15 \pm 4.54$ & 3.22 & - & - & - & - & - & - \\
\hline
\end{tabular}

In Cross I all gene effects (mean, additive, dominance, additivexadditive and dominancexdominance) for days to maturity, number of primary branches panicle ${ }^{-1}$ and number of secondary branches panicle ${ }^{-1}$ were significant except dominance gene effect in days to $50 \%$ flowering and number of panicles plant ${ }^{-1}$. Roy and Senapati (2011) also reported that the dominance effect was not significant for the days to $50 \%$ flowering. In the case of the adequacy of the Simple additive-dominance model, only additive gene effects were significant in panicle length, grain L/B ratio, straw weight (g) and grain yield plant ${ }^{-1}(\mathrm{~g})$. Similarly, only the dominance gene effect was significant in grain breadth $(\mathrm{mm})$. In plant height $(\mathrm{cm})$, number of grains panicle ${ }^{-1}$, number of filled grains panicle ${ }^{-1}$ and harvest index (\%) both additive and dominance gene effects were significant. An additive effect for panicle length and grain length/breadth ratio was reported by Roy and Senapati (2012). Also the importance of additive gene effects were reported by Roy and Senapati (2011), Kacharabhai (2014) and Sultana, et al. (2016). In Cross II all gene effects(mean, additive, dominance, additive $x$ additive and dominancexdominance) for plant height, number of tillers plant ${ }^{-1}$, number of panicles plant ${ }^{-1}$, number of filled grains panicle $e^{-1}$ and fertility percentage were significant except dominance $x$ dominance gene effect in the number of grains panicle ${ }^{-1}$. Similar dominance results were earlier reported for the number of panicles plant ${ }^{-1}$ (Denary et al., 2012), number of tillers plant ${ }^{-1}$ (Kumar et al., 2007) and L/B ratio (Rani et al., 2015). Chaturvedi et al. (2010 and Rao et al., 2017 reported that days to $50 \%$ flowering, $50 \%$ flowering to maturity, plant height, panicle length, grain filling percentage and lengthbreadth ratio were controlled by both additive and dominance gene action. In the case of the adequacy of the simple additive dominance model, only additive gene effect was significant in grain breadth $(\mathrm{mm})$, straw weight $(\mathrm{g})$ and grain yield plant ${ }^{-1}$ (g). Similarly, only dominance gene effect was significant in, days to maturity and number of primary branches panicle ${ }^{-1}$. In a number of secondary branches panicle ${ }^{-1}$, both dominance and additive gene effects were significant. Mather and Jinks (1971) showed that the classification of interactions on the basis of the related magnitudes and signs of the estimates of the five parameters largely depends on the magnitude and signs of the estimates of $h$ and $I$. The opposite sign of dominance (h) and dominancexdominance (I) sign indicated a prevalence of duplicate epistasis whereas complementary epistasis is indicated when signs are the same. In Cross I, days to maturity, number of primary branches panicle ${ }^{-1}$ and number of secondary branches panicle ${ }^{-1}$ showed the duplicate 
type of epistasis whereas, in Cross II plant height, number of filled grains panicle ${ }^{-1}$, number of grains panicle ${ }^{-1}$ and fertility $\%$ showed the duplicate type of epistasis. The duplicate effect of these traits would tend to hinder progress at an increased level of manifestation. Similar results were reported for a number of spikelet panicle ${ }^{-1}$ (Liu and Hong, 2005), for a number of filled grains panicle ${ }^{-1}$ (Kumar et al., 2007), for all the yield and quality related traits like plant height (Roy and Senapati, 2011) and a number of grains panicle ${ }^{-1}$ (Subbulakshmi et al., 2016). In cross 1 the traits like Days to 50\% flowering and number of panicles plant ${ }^{-1}$ showed the presence of a complementary type of epistasis whereas in cross II the complementary type of epistasis was recorded for the number of tillers plant ${ }^{-1}$ and number of panicles plant ${ }^{-1}$. Subbulakshmi et al., 2016 reported the presence of a complementary type of epistasis for days to $50 \%$ flowering. The complementary effect of these traits will produce new recombinants capable of improving yield.

\section{Conclusion}

An additive, dominance, additivexadditive and dominancexdominance interaction effects were present along with either duplicate dominant epistasis or complementary recessive epistasis for most of its contributing traits in Cross I and Cross II. Hence, selection in the early segregating generations might not give desirable recombinants. Pureline selection, heterosis breeding, and reciprocal recurrent selection might be profitable in exploiting additive, dominance, both additive and non-additive gene action to obtain desirable recombinants.

\section{References}

Ajay, B.C., Meena, H.N., Singh, A.L., Dagla, M.C., Kumar, N., Bera, S.K., Gangadhar, K., Makwana, A.D., 2018. Generation mean analysis of yield and mineral nutrient concentrations in peanut (Arachis hypogaea L.). Journal of Oilseeds Research 35(1), 14-20.

Azizi, F., Rezai, A.M., Saedi, G., 2006. Generation means analysis to estimate genetic parameters for different traits in two crosses of corn in bred lines at three planting densities. Journal of Agriculture Science and Technology 8, 153-160.

Cavalli, L.L., 1952. An analysis of linkage in quantitative inheritance. An analysis of linkage in quantitative inheritance.

Chaturvedi, H.P., Talukdar, P., Changkija, S., 2010. Genetic analysis for yield components and yield in rice (Oryza sativa L.). International Journal of Bio-resource and Stress Management 1(1), 48-50.

Denary, E.L., Medhat, E., Dora, S.A., Youssef, M.I.A., Selim, M.S., 2012. Genetic analysis of quantitative traits in two japonica hybrid rice varieties. Journal of Agricultural Research 38(4), 568-586.

Falconer, D.S., 1989. Introduction to quantitative genetics. Longman, Edinburgh.
Gaballah, M.M., Azza, M.M., Milan, S., Hassan, M.M., Marian, B., Abagh, E.L.A., Fayed, M.A., 2020. Genetic diversity of selected rice genotypes under water stress conditions. Plants 10(1), 27.

Gaoh, B.S.B., Gangashetty, P.I., Mohammed, R., Dzidzienyo, D.K., Tongoona, P., 2020. Generation mean analysis of pearl millet (Pennisetum glaucum (L.) R. Br.) grain iron and zinc contents and agronomic traits in West Africa. Journal of Cereal Science 96, 103066.

Giri, R.K., Verma, S.K., Yadav, J.P., 2020. Generation mean analysis for yield and its component traits in diallel population of cotton (Gossypium hirsutum L.). Indian Journal of Agricultural Research 54(6).

Golkar, P., 2018. Inheritance of carthamin and carthamidin in safflower (Carthamus tinctorius L.). Journal of Genetics 97(1), 331-336.

Hayman, B.I., 1958. The separation of epistatic from additive and dominance variation in generation means. Heredity 12, 371-390.

Hayman, B.I., Mather K., 1955. The description of genetic interaction in continuous variation. Biometrics 11, 69-82.

Kacharabhai, P.S., 2015. Genetic analysis of yield and its components traits in rice (Oryza sativa L.). Electronic Journal of Plant Breeding 6(1), 19-25.

Kearsey, M.J., Pooni, H.S., 1996. The genetical analysis of quantitative traits. Chapman and Hall, New York.

Khan, M.H., Dar, Z.A., Dar, S.A., 2015. Breeding strategies for improving rice yield $-A$ review. Agricultural Sciences 6, 467-478.

Kumar, S., Singh, H.B., Sharma, J.K., 2007. Gene action for grain yield, its components and quality traits in hill rice (Oryza sativa L.). Indian Journal of Genetics and Plant Breeding 67(3), 275-277.

Kumar, S.T., Narasimman, R., Eswaran, R., Kumar, C.P.S., Anandan, A., 2007. Studies on genetic variability, heritability and genetic advance in segregating generations of rice (Oryza sativa L.). International Journal of Plant Sciences 2(1), 48-51.

Liu, J.B., Hong, D.L., 2005. Genetic analysis on panicle angle and number of spikelet's per panicle in japonica rice (Oryza sativa). Chinese Journal of Rice Science 19(3), 223-230.

Liu, W., Gowda, M., Steinhoff, J., Maurer, H.P., Wurschum, T., Longin, C.F., Cossic, F., Reif, J.C., 2011. Association mapping in an elite maize breeding population. Theoretical and Applied Genetics 123, 847-858.

Mather, K., 1949. Biometrical genetics. Dover Publications, Inc., New York.

Mather, K., Jinks, J.L., 1971. Biomedical genetics. Cornell University Press.

Naghera, Y.V., Vadodariya, K.V., Patel, R.K., Modha, K.G., Vadodariya, G.D., Vavdiya, P.A., Mungra, K.S., Patel. A.D., 2021. Genetic analysis for seed cotton yield and 
its contributing traits in interspecific crosses of diploid cotton (G. herbaceum $\times$ G. arboreum). Electronic Journal of Plant Breeding 12(2), 563-566.

Panse, V.G., Sukhatme, P.V., 1967. Statistical methods for Agricultural Research Works. III edition, ICAR, New Delhi.

Patel, D.K., Patel, A., Patel, C.J., Jat, A.L., 2021. Generation mean analysis for seed yield and wilt resistance in castor (Ricinus communis L.). Indian Journal of Agricultural Research 1, 4.

Rani, P. J., Satyanarayana, P.V., Chamundeswari, N., Ahamed, M.L., Rani, M.G., 2015. Studies on genetic control of quality traits in rice (Oryza sativa L.) using six parameter model of generation mean analysis. Electronic Journal of Plant Breeding 6(3), 658-662

Rao, M., 2017. Generation mean analysis for grain yield and its component traits in submergence rice. SABRAO Journal of Breeding and Genetics 49(4), 327-335.

Roy, S.K., Senapati, B.K., 2011. Estimation of genetic components for grain yield and quality traits of rice. Oryza 48(1), 22-30.

Roy, S.K., Senapati, B.K., 2012. Combining ability analysis for grain yield and quality characters in rice (Oryza sativa). Indian Journal of Agricultural Sciences 82(4), 293-303.

Said, A.A., 2014. Generation mean analysis in wheat (Triticum aestivum L.) under drought stress conditions. Annals of Agricultural Sciences 59(2), 177-184.
Srinivas, B., Bhadru, D., 2015. Estimation of genetic parameters through generation mean analysis for yield and its components in four upland (Gossypium hirsutum L.) cotton crosses. Electronic Journal of Plant Breeding 6(3), 792-800.

Subbulakshmi, K., Shunmugavalli, N., Muthuswamy, A., 2016. Generation mean analysis for yield and quality traits in $\mathrm{F}_{2}$ and $\mathrm{F}_{3}$ generation of rice (Oryza sativa $\mathrm{L}$.). Electronic Journal of Plant Breeding 7(3), 491-495.

Sultana, R., Ansari, N.A., Ramesha, M.S., Sankar, A.S., Krishna, K.M., 2016. Generation mean analysis of quantitative traits in restorer lines of rice (Oryza sativa L.). Annals of plant and soil research, 586.

Tenorio, F.A., Ye, C., Redona, E., Sierra, S., Laza, M., Argayoso, M.A., 2013. Screening rice genetic resources for heat tolerance. SABRAO Journal of Breeding and Genetics 45(3), 371-381.

Viana, J.M.S., 2005. Dominance, epistasis, heritabilities and expected genetic gains. Genetics and Molecular Biology 28(1), 67-74.

Yadav, H.N., Prasad, R., Singh, S.P., Singh, R.P., Agrawal, R.K., 2013. Detection of epistasis and gene effects for quality and yield traits in rice. Oryza 2, 115-119. 